\title{
Inteligencia emocional rasgo y habilidad como factores asociados al consumo de cannabis en la adolescencia
}

\section{Trait and ability emotional intelligence as factors associated with cannabis use in adolescence}

\author{
Sara González-Yubero*, Raquel Palomera Martín*, Susana lázaro-Visa*. \\ * Universidad de Cantabria, Santander. España.
}

\section{Resumen}

Diversos organismos internacionales alertan sobre el incremento de consumo de cannabis y de su uso extendido entre los adolescentes. El presente estudio ha sido uno de los primeros con el objetivo de analizar el papel de la inteligencia emocional rasgo y habilidad, basada en el modelo de Mayer y Salovey, en relación al consumo de cannabis en adolescentes. En este estudio participaron 799 jóvenes españoles con edades comprendidas entre los 12 y los 16 años. Se administró un autoinforme de inteligencia emocional (IE) rasgo, un test de rendimiento máximo de IE y se preguntó sobre los hábitos relacionados con el consumo de cannabis. Este estudio de tipo transversal se llevó a cabo a través de una metodología de corte cuantitativo y de tipo correlacional. Los principales resultados obtenidos mediante los análisis de regresión una vez controlados el género, la edad y el contexto de centro, revelaron asociaciones negativas entre los factores de comprensión y reparación emocional de la IE rasgo y las variables de consumo de cannabis, al contrario que la atención emocional. Por otro lado, en relación con la IE habilidad, los factores de percepción y facilitación se asociaron de manera inversa al consumo de cannabis en los adolescentes. Los resultados de este estudio sugieren que tanto la IE rasgo como la IE habilidad son constructos complementarios que ayudan a explicar el consumo de cannabis. Estos hallazgos proporcionan evidencias empíricas que podrían orientar intervenciones clínicas y educativas enfocadas a la prevención del consumo en esta etapa. Palabas clave: Inteligencia emocional; autoinforme; test de rendimiento máximo; cannabis; adolescencia.

\begin{abstract}
Many international organisms have warned of the increased consumption of cannabis and its extensive use by adolescents. This study is one of the first with the aim of analyzing the role of ability and trait emotional intelligence, based on the model of Mayer and Salovey, with regards to the consumption of cannabis by adolescents. The study participants were 799 Spanish nationals aged 12 to 16 . They were administered a self-report on trait emotional intelligence (EI), a test of maximum EI performance and were asked about their habits relating to cannabis consumption. This cross-sectional study used a quantitative, correlational methodology. The main results obtained from the regression analysis once gender, age and context of residence were controlled for, revealed negative associations between the factors of understanding and emotional repair of trait EI and the cannabis consumption variables, in contrast to emotional attention. On the other hand, with regards to ability EI, the factors of perception and facilitation were inversely associated with cannabis consumption in adolescents. The results suggest that both trait and ability EI are complementary constructs that help to explain cannabis consumption during this life stage. These findings offer empirical evidence that may help guide clinical and educational interventions focused on prevention of consumption during this period.

Key Words: Emotional intelligence; self-report; performance test; cannabis; adolescence.
\end{abstract}




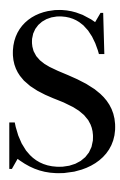

egún el World Drug Report (2018), el uso de cannabis se está incrementando y es la droga más consumida en el mundo por jóvenes y adolescentes a excepción del alcohol y el tabaco. Se estima que unos 17,2 millones $(14,1 \%)$ de jóvenes europeos con edades comprendidas entre los 15 y los 34 años consumieron cannabis en el último año (Observatorio Europeo de las Drogas y las Toxicomanías, 2018). En relación a España, un $31,1 \%$ de los adolescentes entre 14 y 18 años afirma haberlo probado alguna vez, un 26,3\% lo consumió durante el último año y, de estos, un 13,3\% informó de niveles de consumo problemático (Plan Nacional Sobre Drogas, 2018). Así mismo, según la Encuesta sobre Uso de Drogas en Estudiantes de Enseñanzas Secundarias (ESTUDES 2016-2018; Plan Nacional sobre Drogas, 2018), el consumo de cannabis se encuentra más extendido en los varones y la proporción de consumo general aumenta progresivamente con la edad. Investigaciones longitudinales han destacado el consumo de cannabis en el grupo de iguales como uno de los principales factores de riesgo del consumo en los adolescentes (Creemers et al., 2010; von Sydow, Lieb, Pfister, Höfler y Wittchen, 2002). Así mismo, la cantidad y frecuencia de uso de esta sustancia se ha asociado a la aparición de trastornos mentales, abuso y dependencia en adolescentes y jóvenes adultos (Degenhardt et al., 2013; von Sydow et al., 2002). En esta línea, los estudios advierten de que aquellos que inician su consumo a edades tempranas no sólo son más susceptibles de consumir otras sustancias ilegales y desarrollar un patrón de consumo de alcohol de riesgo (Díaz, Busto y Caamaño, 2018; Rial et al., 2018), sino también de una mayor probabilidad de implicarse en conductas relacionadas con el juego problemático (Míguez y Becoña, 2015), presentar problemas escolares y de salud mental, así como de cometer actos de suicidio (Anglin et al., 2012; Silins et al., 2014).

Diversas investigaciones han puesto de manifiesto que la Inteligencia Emocional (IE) entendida como la capacidad para reconocer, comprender y regular las emociones propias y las de los demás, discriminar entre ellas y utilizar la información como guía de los pensamientos y acciones (Mayer y Salovey, 1997), es un predictor de bienestar y de un mejor funcionamiento adaptativo en la adolescencia (Gascó, Badenes y Plumed, 2018; Resurrección, Salguero y Ruiz-Aranda, 2014). La literatura ha dado cuenta del relevante papel que posee la IE en relación con diversos ámbitos de relevancia como la salud mental, psicológica y psicosomática tanto en adultos como en adolescentes (Balluerka, Gorostiaga, Alonso-Arbiol y Aritzeta, 2016; Davis y Humphrey, 2012; Martins, Ramalho y Morin, 2010). Sin embargo, la adolescencia sigue siendo una etapa menos estudiada, lo que plantea un desafío importante para la investigación y el progreso clínico y educativo.

En la actualidad, la literatura científica apoya la distinción de dos constructos de IE que pueden diferenciarse en base al método de medida utilizado para operacionalizarla (Petrides, 2011). La IE rasgo hace referencia a la autopercepción de una serie de aptitudes emocionales evaluadas a través de autoinformes. Por otro lado, la IE habilidad alude a la capacidad para dar respuestas correctas a diversas tareas de corte emocional mediante pruebas de rendimiento máximo. El primer dominio pertenece al ámbito de la personalidad mientras que, el segundo, hace referencia a la capacidad cognitiva y, por consiguiente, su literatura se desarrolla de manera independiente. Mientras que las medidas de autoinforme de IE confían en la percepción de la persona sobre sus propias capacidades emocionales, desde el modelo propuesto por Mayer et al. (1997) se subraya la importancia de emplear medidas de rendimiento máximo para evaluar la habilidad real de la persona, siguiendo la metodología tradicional empleada para valorar las inteligencias cognitivas (Brackett y Salovey, 2006; Mayer, Salovey y Caruso, 2008).

En su trabajo de revisión sobre IE y consumo de sustancias adictivas Kun y Demetrovics (2010) analizaron 36 estudios que, en su mayoría, midieron la IE mediante métodos debidamente probados. Los datos obtenidos en esta investigación apoyan la idea de que bajos niveles de IE rasgo e IE habilidad se relacionan con consumos más problemáticos de alcohol, tabaco y sustancias ilegales en adultos, universitarios y adolescentes. Concretamente, los resultados informaron de que los componentes de percepción y regulación de las emociones presentaron un papel clave en la comprensión del consumo de sustancias adictivas y en las adicciones. Sin embargo, es de resaltar que la mayoría de los estudios utilizaron un índice global de IE y no aportaron información sobre el impacto de cada factor de manera individual, lo que hubiera ayudado a enfocar las intervenciones clínicas y educativas. Además, en una gran parte de los trabajos se aplicaron medidas de autoinforme estudiando únicamente el papel de la IE rasgo. Es por esta razón por la que los autores del estudio recomendaron el uso de medidas conjuntas de IE rasgo e IE habilidad para una comprensión más precisa de esta problemática. Asî mismo, son muy pocas las investigaciones llevadas a cabo en población adolescente y apenas se encuentran estudios que hayan intentado relacionar la IE con el consumo de cannabis en particular.

A continuación, se detallan los hallazgos de las investigaciones que evaluaron la relación entre el consumo de cannabis y la IE rasgo a través del autoinforme Trait Meta-Mood Scale (TMMS; Salovey, Mayer, Goldman, Turvey y Palfai, 1995). En esta línea, Limonero, Tomás-Sábado y Fernández-Castro (2006), observaron como la capacidad percibida para regular los estados emocionales negativos se asoció de manera inversa con el uso habitual de cannabis y tabaco en universitarios. Así mismo, los universitarios con mayor capacidad percibida para comprender con claridad sus estados emocionales informaron de un menor 
consumo de cannabis (Limonero et al., 2006; Limonero, Gómez-Romero, Fernández-Castro y Tomás-Sábado, 2013). Por otro lado, Limonero et al. (2013) comprobaron que la atención emocional junto con el abuso de alcohol fueron los principales factores asociados al abuso de cánnabis. Los autores concluyeron que los jóvenes que atendieron en exceso a sus emociones y carecieron de recursos adecuados para regularlas usaron como mecanismo el consumo excesivo de alcohol que facilitó el abuso de cánnabis. En un estudio con pacientes adultos consumidores de cannabis por prescripción médica, Boden, Gross, Babson y Bonn-Miller (2013), encontraron que una menor capacidad percibida para comprender los estados emocionales daba lugar a una ineficiente regulación emocional, lo que, incrementó el abuso y la dependencia al cannabis. Por otro lado, Ruíz-Aranda et al. (2010), hallaron que los adolescentes que no habían consumido cannabis presentaban una mayor capacidad percibida para regular los estados emocionales negativos y prolongar los positivos que aquellos que afirmaron haberlo consumido.

En dos trabajos llevados a cabo con varones adultos diagnosticados de dependencia al cannabis y grupos control sin problemas de adicción, se evaluó la IE rasgo con The Emotional Intelligence Scale (EIS; Shutte et al., 1998) adaptada por Bhattacharya, Bhattacharya, Dutta y Mandal (2004). En ellos, se encontraron diferencias significativas entre la baja puntuación total de IE y alta alexitimia en los grupos con problemas de dependencia al cannabis en comparación con los grupos control (Nehra, Kumar, Sharma y Nehra, 2013; Nehra et al., 2012). Estos resultados coinciden con la revisión de estudios de Kun et al. (2010), donde se concluye que la decodificación de los estados emocionales es menos precisa en personas con problemas de alcoholismo, fumadores intensivos, usuarios de cannabis y personas con adicción a internet. Del mismo modo, los resultados de investigación de Claros y Sharma (2012), mostraron que niveles bajos en los factores de percepción, uso, comprensión y regulación emocional del EIS se asociaron a un mayor consumo de alcohol y marihuana en adolescentes escolarizados.

Atendiendo a las investigaciones que evaluaron la IE como habilidad mediante pruebas de rendimiento máximo, Brackett, Warner y Mayer (2004), analizaron la relación entre la IE medida a través de Mayer-Salovey-Caruso-Emotional Intelligence Test (MSCEIT; Mayer, Salovey y Caruso, 2002a) y el consumo de drogas legales e ilegales en universitarios. Las puntuaciones de área de este test engloban dos ramas cada una: el área experiencial (percepción y facilitación) que hace referencia a la habilidad para acceder a la información emocional, reconocerla, compararla con otras sensaciones y comprender cómo influye en el pensamiento; y el área estratégica (comprensión y regulación) que hace referencia a la habilidad para comprender y manejar la información de las emociones, tanto propias como ajenas, de valencia positiva o negativa, para adaptarse a las situaciones de la vida diaria de una forma efectiva. Los resultados de su estudio mostraron relaciones negativas significativas entre la puntuación de IE total, el área experiencial y el área estratégica respecto al consumo de marihuana, drogas ilegales, alcohol y consumo con amigos en una muestra de universitarios. En esta línea, Trinidad y Johnson (2002) también encontraron asociaciones negativas entre los factores de percepción, comprensión, regulación emocional e IE total evaluada a través de Multifactor Emotional Intelligence Scale (MEIS; Mayer, Salovey y Caruso, 1997) y el consumo de alcohol y tabaco en población adolescente. Los autores concluyeron que la IE fue un factor protector del consumo que, además, redujo la influencia ejercida por el grupo de iguales para consumir.

Como se puede observar, y a pesar de la importancia de los hallazgos previos, la mayor parte de las investigaciones fueron realizadas con población clínica adulta y universitaria, siendo apenas inexistentes los trabajos que analizan la implicación de la IE y el consumo de cannabis en la etapa adolescente. Así mismo, apenas se encuentran investigaciones que combinen la evaluación de los factores de IE rasgo e IE habilidad aportando una comprensión completa de su papel sobre el consumo de esta sustancia.

Dada la preocupación que origina el consumo abusivo de cannabis a edades tempranas, este trabajo se plantea con el objetivo de analizar la relación de las dimensiones de la IE rasgo y habilidad sobre diversas variables de consumo de cannabis en adolescentes de 12 a 16 años.

En base a los resultados de estudios previos se formula la siguiente hipótesis de trabajo: las dimensiones de la IE rasgo e IE habilidad estarán asociadas de manera inversa y significativa a las variables dependientes de consumo de cannabis («consumo de hachís o marihuana alguna vez»; «número de días de consumo de hachís o marihuana en los últimos 12 meses»; «cantidad de unidades semanales consumidas»; «consumo de hachís o marihuana por ofrecimiento de un amigo»), a excepción del factor de atención a las propias emociones de IE rasgo, que se asociará de manera positiva y significativa con las mismas. Ambos constructos serán factores explicativos complementarios en la comprensión de esta problemática.

\section{Método}

\section{Tipo de estudio}

Este estudio presenta un diseño de tipo transversal con una metodología cuantitativa de tipo correlacional.

\section{Participantes}

En este estudio participaron un total de diez centros de Educación Secundaria Obligatoria de la comunidad de Cantabria. Se llevó a cabo un muestreo aleatorio estratificado en función de la titularidad del centro escolar (público 
o privado/concertado) y la localización en entorno rural o urbano, siguiendo la proporción presente en la población de referencia. Cabe señalar que se obtuvo una participación del $66,6 \%$ de los centros educativos seleccionados inicialmente debido a la alta carga de trabajo que algunos presentaron. La muestra inicial fue de 844 participantes. El criterio de exclusión fue encontrarse fuera del rango de edad objeto de estudio 12-16 años $(\mathrm{N}=21)$, así como no haber rellenado el cuestionario completo al finalizar la segunda sesión $(\mathrm{N}=24)$. La muestra final fue del 94,6\% respecto a la muestra inicial $(\mathrm{N}=799)$ y estuvo conformada por escolares de edades comprendidas entre los 12 y 16 años $(M=14,49 ; \mathrm{DT}=1,17)$ con una distribución equilibrada por género ( $51,8 \%$ mujeres y $48,2 \%$ hombres). Un $51,4 \%$ pertenecieron a centros privados/concertados y un $48,6 \%$ a públicos, localizados en el medio urbano (64\%) y rural $(36 \%)$.

\section{Instrumentos}

\section{Variables dependientes:}

- Cuestionario de consumo de cannabis. Se realizó una adaptación de 4 ítems de ESTUDES (Plan Nacional sobre Drogas, 2018). En primer lugar, se preguntó por el «consumo de hachís o marihuana alguna vez» (sí he consumido/no he consumido), así como por el «consumo de hachís o marihuana por ofrecimiento de un amigo» (si consumo/no consumo). Por otro lado, las variables que se describen a continuación se dicotomizaron en función de la mediana para introducirlas en los análisis posteriores: «número de días de consumo de hachís o marihuana en los últimos 12 meses» (<40 días; $>=40$ días) y «cantidad de unidades semanales consumidas» (<10 unidades; $>=10$ unidades).

\section{Variables independientes:}

- Cuestionario de datos sociodemográficos. Se recogió el género (mujer/hombre), la edad (12-13 años/14-16 años) y el contexto de centro (urbano/rural).

- Trait Meta-Mood Scale (TMMS; Salovey et al., 1995, validado en población adolescente española por Salguero, Fernández-Berrocal, Balluerka y Aritzeta, 2010). Este autoinforme está integrado por 24 ítems y proporciona un indicador de los niveles de inteligencia emocional rasgo. Sus ítems se evalúan a través de una escala tipo Likert de 5 puntos desde «Muy en desacuerdo» (1) hasta «Muy de acuerdo» (5). Está compuesto por tres subescalas: atención a los sentimientos es el grado en el que las personas creen prestar atención a sus emociones y sentimientos (p. ej., "presto mucha atención a los sentimientos»); claridad emocional se refiere al grado en que las personas creen comprender sus estados emocionales (p. ej., «frecuentemente me equivoco con mis sentimientos»); y, por último, reparación de las emociones alude a la creencia de la persona en su capacidad para interrumpir y regular estados emocionales negati- vos y prolongar los positivos (p. ej., «aunque a veces me siento triste, suelo tener una visión optimista»). Cada subescala está formada por 8 items y no ofrece una puntuación global. El autoinforme original ha demostrado una alta consistencia interna (alpha de Cronbach para Atención $=, 90$, Claridad $=, 90$, Reparación $=, 86)$. Los alphas de Cronbach obtenidos para este estudio fueron de ,87 para Atención, ,85 para Claridad y ,82 para Reparación.

- Test de Inteligencia Emocional de la Fundación Botín para Adolescentes (TIEFBA; Fernández-Berrocal, Ruiz-Aranda, Salguero, Palomera y Extremera, 2011). Esta batería autoadministrada dirigida a adolescentes (12-17 años) proporciona medidas de los niveles de rendimiento en cada una de las cuatro habilidades emocionales del modelo teórico de Mayer y Salovey (1997). El test está compuesto por 143 ítems que plantean situaciones emocionales a través de ocho historietas breves con personajes. Proporciona un total de 7 puntuaciones: dos de área, cuatro de rama y la puntuación total.

En este estudio se han utilizado las cuatro puntuaciones de rama que hacen referencia a cada una las cuatro habilidades de IE con el objetivo de obtener resultados más concisos que ayuden en futuras intervenciones. Percepción emocional hace referencia al grado en el que las personas pueden identificar de forma adecuada sus propias emociones y las de los demás, así como los estados y sensaciones fisiológicas y cognitivas que éstas conllevan. Implica la capacidad para etiquetar y otorgarle una palabra adecuada a las emociones, ya sean positivas tales como la alegría, el amor o la gratitud, o negativas como la ira, la tristeza o la envidia (p. ej., «¿en qué medida crees que muestra Rocío cada uno de los siguientes sentimientos?»); facilitación emocional es la habilidad para acceder a las emociones y sentimientos y para generarlos con el objetivo de facilitar el pensamiento, los procesos cognitivos o la resolución de problemas entre otros (p. ej., "¿en qué medida sentirse así le ayudará a Rocío a revisar la lista de materiales escolares que debe comprar este año?»); comprensión emocional incluye la habilidad para interpretar el significado de las emociones complejas, por ejemplo, las generadas durante una situación interpersonal, así como la destreza para reconocer las transiciones de unos estados emocionales a otros y la aparición de sentimientos simultáneos (p. ej., "¿qué puede estar pensando Rocío para sentirse así?»); regulación emocional incluye la habilidad para regular las emociones propias y ajenas, moderando las emociones negativas e intensificando las positivas sin reprimir ni exagerar la información que comunican (p. ej., «¿qué puede hacer Rocío para irse contenta al colegio?»). Este test ha demostrado una consistencia interna adecuada con valores alpha de Cronbach de ,86 para Percepción, ,76 para Facilitación, ,76 para Comprensión y ,74 para 
Regulación. Los alphas de Cronbach obtenidos para este estudio fueron de ,86 para Percepción, ,78 para Facilitación, ,80 para Comprensión y ,76 para Regulación.

\section{Procedimiento}

En primer lugar, se emitió un comunicado a los centros educativos solicitando la autorización firmada de las familias y el consentimiento informado de los estudiantes. Para garantizar el anonimato durante el proceso se utilizaron códigos numéricos en cada cuestionario evitando la identificación personal de los alumnos. Por otro lado, se administró un autoinforme de IE rasgo, un test de rendimiento máximo de IE y se preguntó a los estudiantes sobre los hábitos relacionados con el consumo de cannabis. Un investigador estuvo presente en el aula durante las dos sesiones no consecutivas de 45 minutos en las que se realizó la prueba en papel y a bolígrafo.

\section{Consideraciones éticas}

Esta investigación se rige según los principios recogidos en la Declaración de Helsinki (World Medical Association, 2013). Así mismo, el desarrollo del plan de investigación del presente estudio fue presentado a la Comisión Académica de Estudios de Doctorado de la Universidad de Cantabria.

\section{Análisis de datos}

Para el procesamiento de análisis de datos se empleó el paquete estadístico SPSS Statistics 24.0. El estudio se llevó a cabo a través de una metodología de corte cuantitativo y tipo correlacional. En primer lugar, se calcularon los índices de fiabilidad alpha de Cronbach para cada uno de los factores de IE evaluados. Se realizaron análisis descriptivos y de correlación biserial puntual de las variables de estudio. Posteriormente, se construyeron modelos de regresión binomial a partir de los factores de IE rasgo (Atención, Claridad y Reparación emocional) e IE habilidad (Percepción, Facilitación, Comprensión y Regulación emocional), teniendo además en cuenta el efecto de la edad, el género y el contexto de centro a la hora de observar su asociación con las variables dependientes: «consumo de hachís o marihuana alguna vez»; «número de días de consumo de hachís o marihuana en los últimos 12 meses»; «cantidad de unidades semanales consumidas»; «consumo de hachís o marihuana por ofrecimiento de un amigo»). Así mismo se obtuvieron las Razones de Prevalencia (RP) con sus respectivos Intervalos de Confianza al 95\% (IC95\%) (Espelt, Bosque-Prous y Marí-Dell'Olmo, 2019; Espelt, Mari-Dell'Olmo, Penelo y Bosque-Prous, 2017). Con objeto de sintetizar la cantidad de datos, únicamente se presentan los modelos finales que explicaron un mayor porcentaje de varianza.

\section{Resultados}

Prevalencia del consumo de cannabis en los adolescentes

En la Tabla 1 se presentan los datos más significativos de la prevalencia del consumo de cannabis en la muestra de estudio. Como se puede apreciar, un primer dato de interés es que 2 de cada 10 adolescentes consumieron cannabis. Entre estos, una mayoría lo hizo durante cuarenta días o más en los últimos 12 meses (74,9\%). Aproximadamente la mitad de los consumidores fumaron diez o más unidades semanales. Así mismo, una quinta parte afirmó que consumiría ante el ofrecimiento de los amigos (20,5\%). Por otro lado, en relación con las variables sociodemográficas se observa que un $18,3 \%$ de las mujeres consumieron cannabis alguna vez, mientras que en los hombres la prevalencia fue algo superior (26,4\%). Respecto al grupo de edad 12-13 años, un 13,9\% afirmó haber consumido esta sustancia, siendo apreciable un incremento del consumo en el grupo de 14-16 años (27,3\%). En relación al contexto de centro, 2 de cada 10 estudiantes de centro urbano consumieron cannabis, mientras que su uso fue algo más elevado en los estudiantes pertenecientes a centros de entorno rural $(28,1 \%)$.

\section{Análisis descriptivos y de correlación entre las varia- bles de consumo de cannabis, la IE rasgo y habilidad}

A continuación, se muestran los estadísticos descriptivos de las subescalas de IE utilizadas en este estudio (Tabla 2). Por otro lado, se detallan los resultados del análisis de correlación entre las variables de IE y las de consumo de cannabis (Tabla 3). Como se puede apreciar, se observan asociaciones negativas significativas entre las variables de IE rasgo e IE habilidad y el consumo de cannabis a excepción de la atención a los propios sentimientos que correlacionó de manera directa y significativa. De forma específica, para la variable de consumo de «cannabis alguna vez», las correlaciones más elevadas se encontraron con los factores de percepción y facilitación emocional. En relación con la «frecuencia de consumo el último año» destacaron las correlaciones con los factores de atención y reparación emocional. Respecto a las «unidades de consumo semanal» y «consumo por ofrecimiento de los amigos», las correlaciones más elevadas se obtuvieron de nuevo con percepción y facilitación emocional.

En este apartado y, con objeto de sintetizar la abundante cantidad de datos, únicamente se presentan los cuatro modelos finales que resultaron estadísticamente significativos y explicaron un mayor porcentaje de la varianza criterio

\section{Regresión binomial para el cálculo de las razones de prevalencia del consumo de cannabis en función de las variables de IE rasgo y habilidad teniendo en cuenta el género, la edad y el contexto de centro escolar}

En primer lugar, en relación al modelo creado para la variable dependiente «consumo de cannabis alguna vez» 
Tabla 1. Prevalencias de consumo de cannabis.

\begin{tabular}{|c|c|c|c|}
\hline Cannabis & Categorías de respuesta & Porcentaje & $\mathbf{N}$ \\
\hline \multirow{2}{*}{ Consumo de cannabis } & Sí he consumido & 22,3 & 179 \\
\hline & No he consumido & 77,7 & 620 \\
\hline \multirow{2}{*}{ Consumo último año } & $>=40$ días & 74,9 & 134 \\
\hline & < 40 días & 25,1 & 45 \\
\hline Cantidad unidades semanales & $>=10$ & 51,4 & 92 \\
\hline \multirow{2}{*}{ Consumo ante el ofrecimiento de amigos } & Sí consumiría & 20,5 & 161 \\
\hline & No consumiría & 79,5 & 638 \\
\hline \multirow{4}{*}{ Consumo por género } & Mujer si & 18,3 & 73 \\
\hline & Mujer no & 81,8 & 327 \\
\hline & Hombre si & 26,4 & 106 \\
\hline & Hombre no & 73,6 & 293 \\
\hline \multirow{4}{*}{ Consumo por edad } & 12-13 años si & 13,9 & 41 \\
\hline & $12-13$ años no & 73,7 & 255 \\
\hline & 14-16 años si & 27,3 & 137 \\
\hline & 14-16 años no & 58,9 & 366 \\
\hline \multirow{4}{*}{ Consumo por municipio de centro } & Urbano si & 19,6 & 107 \\
\hline & Urbano no & 80,4 & 438 \\
\hline & Rural si & 28,1 & 71 \\
\hline & Rural no & 71,9 & 183 \\
\hline
\end{tabular}

Tabla 2. Descriptivos de las variables de inteligencia emocional rasgo y habilidad en función del consumo o no consumo de cannabis.

\begin{tabular}{|c|c|c|c|c|c|c|}
\hline Consume & & Min/Max & M & Me & DT & IC 95\% \\
\hline \multirow{2}{*}{ TMMS Atención } & No & $8 / 40$ & 23,80 & 23,00 & 7,20 & $23,20 / 24,30$ \\
\hline & $\mathrm{Si}$ & $10 / 40$ & 28,30 & 30,00 & 7,80 & $27,20 / 29,50$ \\
\hline \multirow{2}{*}{ TMMS Claridad } & No & $8 / 40$ & 23,70 & 24,00 & 7,20 & $23,10 / 24,30$ \\
\hline & $\mathrm{Si}$ & $8 / 40$ & 18,70 & 16,00 & 7,10 & $17,70 / 19,80$ \\
\hline \multirow{2}{*}{ TMMS Reparación } & No & $10 / 40$ & 26,10 & 26,00 & 7,00 & $25,50 / 26,60$ \\
\hline & $\mathrm{Si}$ & $10 / 40$ & 20,40 & 18,00 & 7,30 & $19,30 / 21,50$ \\
\hline \multirow{2}{*}{ TIEFBA Percepción } & No & $75 / 129$ & 103,00 & 105,00 & 12,40 & $101,90 / 103,90$ \\
\hline & $\mathrm{Si}$ & $76 / 123$ & 90,80 & 90,20 & 11,10 & 89,00 / 92,60 \\
\hline \multirow{2}{*}{ TIEFBA Facilitación } & No & $72 / 139$ & 107,20 & 108,00 & 14,00 & $106,00 / 108,40$ \\
\hline & $\mathrm{Si}$ & $73 / 123$ & 94,40 & 94,70 & 11,80 & $92,60 / 96,20$ \\
\hline \multirow{2}{*}{ TIEFBA Comprensión } & No & $77 / 144$ & 106,50 & 106,40 & 14,00 & $105,40 / 107,70$ \\
\hline & $\mathrm{Si}$ & $74 / 134$ & 95,30 & 94,40 & 13,00 & $91,90 / 93,30$ \\
\hline \multirow{2}{*}{ TIEFBA Regulación } & No & $70 / 114$ & 92,60 & 92,90 & 8,80 & $93,30 / 97,30$ \\
\hline & $\mathrm{Si}$ & $70 / 111$ & 86,70 & 84,90 & 8,00 & $85,50 / 87,90$ \\
\hline
\end{tabular}

Nota. TMMS: Trait Meta Mood Scale; TIEFBA: Test de Inteligencia Emocional de la Fundación Botín para Adolescentes. M: media; Me: mediana; DT: desviación típica; IC: intervalo de confianza.

Tabla 3. Correlación biserial-puntual entre las variables de IE rasgo y habilidad y el consumo de cannabis.

\begin{tabular}{|c|c|c|c|c|}
\hline & Alguna vez & Último año & Unidades semanales & Consumo ante ofrecimiento amigos \\
\hline 1. TMMS - Atención &, $25 * \star$ &, $26^{\star \star}$ & ,08 &, $29 * \star$ \\
\hline 2. TMMS - Claridad &,$- 28^{\star \star}$ &,$- 23^{\star \star}$ &,$- 21^{\star \star}$ &,$- 30^{\star \star}$ \\
\hline 3. TMMS - Reparación &,$- 32^{\star \star}$ &,$- 24^{\star \star}$ &,- 10 &,$- 33^{\star *}$ \\
\hline 4. TIEFBA - Percepción &,$- 38^{\star \star}$ &,$- 17^{\star}$ &,$- 25^{\star \star}$ &,$- 42^{\star \star}$ \\
\hline 5. TIEFBA - Facilitación &,$- 37^{\star \star}$ &,$- 22^{\star \star}$ &,$- 21^{\star \star}$ &,$- 34^{\star \star}$ \\
\hline 6. TIEFBA - Compresión &,$- 33^{\star \star}$ &,- 08 &,$- 20^{\star \star}$ &,$- 30^{\star \star}$ \\
\hline 7. TIEFBA - Regulación &,$- 28^{\star \star}$ &,- 08 &,- 12 &,$- 27^{\star \star}$ \\
\hline
\end{tabular}

Nota. TMMS: Trait Meta Mood Scale; TIEFBA: Test de Inteligencia Emocional de la Fundación Botín para Adolescentes.

${ }^{\star}=\mathrm{p}<, 05{ }^{\star \star}=\mathrm{p}<, 01$. 
entraron a formar parte de la ecuación las variables independientes de claridad, reparación, percepción, facilitación emocional y edad. De entre ellas, la mayores RP se encontraron para los factores de claridad $[\mathrm{RP}=0,96$; IC95\% $(0,92-0,99)]$ y facilitación emocional $[\mathrm{RP}=0,96$; IC95\% $(0,94-0,97)]$, siendo estas similares a las de percepción emocional $[\mathrm{RP}=0,95 ; \mathrm{IC95 \%}(0,94-0,97)]$ y reparación $[\mathrm{RP}=0,93$; IC95\%(0,90-0,97)]. Finalmente, los casos de 14 a 16 años presentan una prevalencia de consumo de 0,73 veces mayor que la de los sujetos de 12-13 años. En segundo lugar, cuando tomamos como variable criterio el «consumo de cannabis al año» entraron a formar parte de la ecuación las variables independientes de reparación $[\mathrm{RP}=0,97 ; \mathrm{IC95 \%}(0,96-0,98)]$ y facilitación emocional $[\mathrm{RP}=0,98$; IC95\% $(0,97-0,99)]$. Para la variable dependien- te «cantidad de unidades semanales», entraron a formar parte de la ecuación claridad $[\mathrm{RP}=0,96 ; \operatorname{IC95} \%(0,93-0,99)]$ y percepción emocional $[\mathrm{RP}=0,97 ; \operatorname{IC95\% }(0,96-0,98)]$. Finalmente, para el modelo de la variable dependiente «consumo por ofrecimiento de los amigos» entraron a formar parte de la ecuación las variables independientes de atención, claridad, reparación, percepción, facilitación y edad. De entre ellas, las mayores RP se encontraron para los factores de atención $[\mathrm{RP}=1,03$; IC95\% (1,00-1,07)] y facilitación [RP=0,97; IC95\% (0,95-0,99)] seguido de reparación $[\mathrm{RP}=0,94 ; \operatorname{IC} 95 \%(0,91-0,98)]$ y percepción emocional $[\mathrm{RP}=0,93$; IC95\%(0,91-0,95)]. Los casos de 14 a 16 años presentaron una prevalencia de consumo de 0,65 veces mayor que la de los sujetos de 12-13 años.

Tabla 4. Regresión Binomial del consumo de cannabis en función de la IE rasgo y habilidad junto a los factores sociodemográficos.

\begin{tabular}{|c|c|c|c|c|c|}
\hline & B & E.T. & Wald & $\mathbf{R P}$ & IC $95 \%$ de la OR \\
\hline \multicolumn{6}{|l|}{ Consumo alguna vez } \\
\hline TMMS - Claridad & $-0,07$ & 0,00 & $49,52^{\star \star}$ & 0,96 & $0,92 / 0,99$ \\
\hline TMMS - Reparación & $-0,08$ & 0,00 & $61,15^{\star \star}$ & 0,93 & $0,90 / 0,97$ \\
\hline TIEFBA - Percepción & $-0,06$ & 0,00 & $94,58^{\star \star}$ & 0,95 & $0,94 / 0,97$ \\
\hline TIEFBA - Facilitación & $-0,04$ & 0,00 & $92,12^{\star \star}$ & 0,96 & $0,94 / 0,97$ \\
\hline Género (M / H) & 0,18 & 0,22 & 0,42 & 0,93 & $0,83 / 1,15$ \\
\hline Edad (12-13 / 14-16) & 0,48 & 0,24 & $0,05^{\star}$ & 0,73 & $0,63 / 1,00$ \\
\hline Contexto (R / U) & 0,20 & 0,23 & 0,39 & 0,93 & $0,83 / 1,14$ \\
\hline \multicolumn{6}{|l|}{ Consumo último año } \\
\hline TMMS - Reparación & $-0,02$ & 0,01 & $8,09 * \star$ & 0,97 & $0,96 / 0,98$ \\
\hline TIEFBA - Facilitación & $-0,01$ & 0,00 & $3,76^{\star}$ & 0,98 & 0,97 / 0,99 \\
\hline Género $(M / H)$ & $-0,25$ & 0,24 & 1,03 & 0,32 & $0,44 / 1,30$ \\
\hline Edad (12-13 / 14-16) & 0,48 & 0,27 & 2,61 & 1,60 & $0,88 / 2,82$ \\
\hline Contexto (R / U) & 0,19 & 0,30 & 0,51 & 1,22 & $0,70 / 2,26$ \\
\hline \multicolumn{6}{|c|}{ Cantidad de unidades semanales } \\
\hline TMMS - Claridad & $-0,04$ & 0,02 & $6,84^{\star \star}$ & 0,96 & $0,93 / 0,99$ \\
\hline TIEFBA - Percepción & $-0,02$ & 0,01 & $8,31^{\star \star}$ & 0,97 & $0,96 / 0,98$ \\
\hline Género $(M / H)$ & 0,06 & 0,15 & 0,15 & 1,07 & 0,77 / 1,46 \\
\hline Edad (12-13 / 14-16) & 0,32 & 0,20 & 2,15 & 1,39 & $0,90 / 2,14$ \\
\hline Contexto (R / U) & 0,03 & 0,15 & 0,03 & 1,03 & $0,76 / 1,38$ \\
\hline \multicolumn{6}{|c|}{ Consumo ante el ofrecimiento de amigos } \\
\hline TMMS - Atención & 0,08 & 0,00 & $51,15^{\star \star}$ & 1,03 & $1,00 / 1,07$ \\
\hline TMMS - Claridad & $-0,09$ & 0,00 & $55,53^{\star \star}$ & 0,96 & 0,92 / 0,99 \\
\hline TMMS - Reparación & $-0,09$ & 0,00 & $62,89^{\star \star}$ & 0,94 & $0,91 / 0,98$ \\
\hline TIEFBA - Percepción & $-0,07$ & 0,00 & $115,33^{\star \star}$ & 0,93 & $0,91 / 0,95$ \\
\hline TIEFBA - Facilitación & $-0,04$ & 0,00 & $67,18^{\star \star}$ & 0,97 & $0,95 / 0,99$ \\
\hline Género $(M / H)$ & 0,09 & 0,23 & 0,18 & 0,96 & $0,86 / 1,21$ \\
\hline Edad (12-13 /14-16) & 0,55 & 0,27 & $3,92^{\star}$ & 0,65 & $0,53 / 0,99$ \\
\hline Contexto (R / U) & 0,08 & 0,22 & 0,09 & 0,97 & $0,84 / 1,29$ \\
\hline
\end{tabular}

Nota. TMMS: Trait Meta Mood Scale; TIEFBA: Test de Inteligencia Emocional de la Fundación Botín para Adolescentes; B: coeficiente; E.T.: error estándar; RP = Razón de prevalencias; IC: intervalo de confianza.

$*=p<, 05 ;{ }^{* *}=p<, 01$. 
Regresión binomial del consumo de cannabis en función de la IE rasgo y habilidad para cada grupo de edad (12-13 años y 14-16 años)

Como se puede apreciar anteriormente (Tabla 4), la variable sociodemográfica edad resultó significativa para el modelo de «consumo de cannabis alguna vez», así como para el modelo de «consumo por ofrecimiento de los amigos». A continuación, se estratifican los resultados en función de los dos grupos de edad para ambas variables de consumo (Tablas 5 y 6 ). En primer lugar, para el modelo de «consumo de cannabis alguna vez» con el grupo de 12 a 13 años entraron a formar parte de la ecuación las variables independientes reparación, percepción y facilitación. De entre ellas, la mayores RP se encontraron para los factores de facilitación emocional [RP=0,97; IC95\% $(0,98-0,99)]$, seguido de percepción $[\mathrm{RP}=0,96$; IC95\% $(0,93-0,99)]$ y reparación $[\mathrm{RP}=0,93$; IC95\% $(0,89-0,97)]$. Así mismo, para el modelo del grupo de 14 a 16 años entraron a formar parte de la ecuación las variables independientes atención, reparación, percepción y facilitación. Las mayores RP se encontraron para atención [RP=1,07; IC95\% $(1,04-1,09)]$ y facilitación $[\mathrm{RP}=0,96 ; \mathrm{IC} 95 \%(0,95-0,97)]$, seguido de percepción $[\mathrm{RP}=0,95 ; \operatorname{IC} 95 \%(0,94-0,96)]$ y reparación
$[\mathrm{RP}=0,93$; IC95\% $(0,90-0,95)]$. En segundo lugar, para el modelo de la variable dependiente "consumo por ofrecimiento de los amigos» entraron a formar parte de la ecuación las variables independientes reparación $[\mathrm{RP}=0,92$; IC95\% $(0,87-0,97)]$ y percepción $[\mathrm{RP}=0,94$; $\operatorname{IC95\% }(0,92-$ $0,97)]$. Finalmente, para el grupo de edad de 14 a 16 años entraron a formar parte de la ecuación las variables atención, claridad, percepción y facilitación. De entre ellas, la mayores $\mathrm{RP}$ se encontraron para atención $[\mathrm{RP}=1,07$; IC95\% (1,05-1,09)] y facilitación [RP=0,96; IC95\% $(0,95$ $0,97)]$, seguido de percepción $[\mathrm{RP}=0,94$; IC95\% $(0,92-$ $0,96)]$ y claridad $[\mathrm{RP}=0,93 ; \mathrm{IC} 95 \%(0,90-0,95)]$.

\section{Discusión}

Los resultados obtenidos en el presente estudio muestran que las prevalencias de consumo de cannabis en escolares son coincidentes en líneas generales con las de la última Encuesta sobre el Uso de Drogas en Estudiantes de Enseñanzas Secundarias (ESTUDES 2016-2018) (Plan Nacional sobre Drogas, 2018), algo que posee importantes implicaciones a nivel de prevención y exalta la necesidad del trabajo intensivo al inicio de la Educación Secundaria

Tabla 5. Regresión Binomial del consumo de cannabis en función de la IE rasgo y habilidad, segmentado para casos con 12-13 años de edad.

\begin{tabular}{|c|c|c|c|c|c|}
\hline & B & E.T. & Wald & $\mathbf{R P}$ & IC $95 \%$ de la OR \\
\hline \multicolumn{6}{|l|}{ Consumo alguna vez } \\
\hline TMMS - Reparación & $-0,07$ & 0,02 & $11,64^{\star *}$ & 0,93 & $0,89 / 0,97$ \\
\hline TIEFBA - Percepción & $-0,04$ & 0,01 & $8,39^{\star \star}$ & 0,96 & $0,93 / 0,99$ \\
\hline TIEFBA - Facilitación & $-0,02$ & 0,01 & $4,26^{*}$ & 0,97 & $0,98 / 0,99$ \\
\hline \multicolumn{6}{|c|}{ Consumo ante el ofrecimiento de amigos } \\
\hline TMMS - Reparación & $-0,08$ & 0,03 & $9,23^{\star \star}$ & 0,92 & $0,87 / 0,97$ \\
\hline TIEFBA - Percepción & $-0,06$ & 0,01 & $18,23^{\star \star}$ & 0,94 & $0,92 / 0,97$ \\
\hline
\end{tabular}

Nota. TMMS: Trait Meta Mood Scale; TIEFBA: Test de Inteligencia Emocional de la Fundación Botín para Adolescentes; B: coeficiente; E.T.: error estándar; RP: Razón de prevalencias; IC: intervalo de confianza.

$\star=p<, 05 ;{ }^{\star *}=p<, 01$.

Tabla 6. Regresión Binomial del consumo de cannabis en función de la IE rasgo y habilidad, segmentado para casos con 14-15-16 años de edad.

\begin{tabular}{|c|c|c|c|c|c|}
\hline & B & E.T. & Wald & $\mathbf{R P}$ & IC $95 \%$ de la OR \\
\hline \multicolumn{6}{|l|}{ Consumo alguna vez } \\
\hline TMMS - Atención & 0,06 & 0,01 & $33,32^{\star *}$ & 1,07 & $1,04 / 1,09$ \\
\hline TMMS - Reparación & $-0,08$ & 0,01 & $42,88^{\star \star}$ & 0,93 & $0,90 / 0,95$ \\
\hline TIEFBA - Percepción & $-0,05$ & 0,00 & $63,81^{\star \star}$ & 0,95 & $0,94 / 0,96$ \\
\hline TIEFBA - Facilitación & $-0,04$ & 0,00 & $82,40 \star \star$ & 0,96 & $0,95 / 0,97$ \\
\hline \multicolumn{6}{|c|}{ Consumo ante el ofrecimiento de amigos } \\
\hline TMMS - Atención & 0,07 & 0,01 & $33,13^{\star \star}$ & 1,07 & $1,05 / 1,09$ \\
\hline TMMS - Claridad & $-0,08$ & 0,01 & $36,18^{\star \star}$ & 0,93 & $0,90 / 0,95$ \\
\hline TIEFBA - Percepción & $-0,06$ & 0,01 & $80,95^{\star \star}$ & 0,94 & $0,92 / 0,96$ \\
\hline TIEFBA - Facilitación & $-0,04$ & 0,00 & $56,50 * \star$ & 0,96 & $0,95 / 0,97$ \\
\hline
\end{tabular}


Obligatoria e incluso desde los últimos cursos de Educación Primaria.

El objetivo principal de esta investigación consistió en analizar la relación entre la IE rasgo, la IE habilidad y el consumo de cannabis en adolescentes escolarizados teniendo en cuenta el género, la edad y el contexto de centro. Los hallazgos de este estudio aportan indicios sobre el papel de la IE en relación al consumo de cannabis a edades tempranas. Así mismo, es de resaltar que ambos constructos de IE fueron componentes explicativos complementarios de esta problemática. En este sentido, tanto las dimensiones de la IE rasgo, como el área experiencial de IE que incluye la habilidad para percibir y usar las emociones facilitando la toma de decisiones y el rendimiento cognitivo, se mostraron asociadas al consumo de cannabis en los adolescentes. Sin embargo, el área estratégica que hace referencia a la habilidad para comprender el significado de las emociones y utilizarlo para conseguir un objetivo determinado, no resultó explicativo del uso de esta sustancia. Por otro lado, es destacable la influencia de la atención emocional sobre el consumo conforme aumenta la edad de los adolescentes.

A partir de los datos obtenidos y, en relación con los tres factores de IE rasgo, se puede decir que los adolescentes con una mayor puntuación en atención a sus propias emociones presentaron más probabilidades de consumir ante el ofrecimiento del grupo de amigos. La atención emocional también resultó una de las más asociadas al consumo de cannabis alguna vez y al consumo por ofrecimiento de los amigos en el grupo de 14-16 años de edad. En este contexto diversas investigaciones han señalado la implicación entre elevados niveles de atención emocional y abuso de alcohol, tabaco y cannabis en adultos y adolescentes (Limonero et al., 2013; Ruiz-Aranda et al., 2010, Ruíz-Aranda et al., 2006). La investigación ha demostrado asociaciones negativas consistentes entre los factores de claridad y reparación emocional respecto a la ansiedad y depresión en población adolescente (Fernández-Berrocal, Alcaide, Extremera y Pizarro, 2006; Extremera y Fernández-Berrocal, 2006; Lombas, Martín-Albo, Valdivia-Salas y Jiménez, 2014). Así mismo, mientras que una mayor claridad y reparación se han relacionado con el uso adecuado de estrategias de manejo del estrés (Fernández, Velasco y Campos, 2003; Saklofske, Austin, Galloway y Davidson, 2007), la atención emocional ha mostrado asociaciones positivas con la ansiedad, depresión y estrategias de afrontamiento desajustadas como el pensamiento rumiativo en los jóvenes (Extremera y Fernández-Berrocal, 2006; Lombas et al., 2014; Saklofske et al., 2007). En este sentido, la tendencia a focalizar la atención a los estados emocionales propios permite seguir el proceso de nuestras emociones, pero esto puede no resultar adaptativo en algunas ocasiones. Altos niveles de atención a los estados emocionales podrían provocar un incremento del pensamiento rumia- tivo y un estado de ánimo displancetro (Ruíz-Aranda et al., 2006). Si el adolescente focaliza una atención elevada en sus sentimientos, pero no es capaz de clarificar qué siente en cada momento ni de regular internamente sus emociones, es más probable que opte por consumir para mitigar los estados emocionales aversivos. La búsqueda de identidad personal, el alejamiento de los valores familiares y la necesidad de aceptación por el grupo son características evolutivas que se ven acentuadas con la edad (Sussman, Unger y Dent, 2004), lo que podría explicar el hecho de que la atención emocional se convierta en un facilitador del consumo de cannabis y de su uso ante el ofrecimiento de los iguales en el grupo de edad más avanzada (14 a 16 años).

Por otro lado, un bajo nivel de claridad y reparación emocional de IE rasgo se asoció de forma negativa con el consumo de cannabis alguna vez y con una mayor probabilidad de fumar por ofrecimiento de los amigos. De igual modo, una menor capacidad percibida para comprender con claridad los estados emocionales se relacionó con mayores cantidades de consumo semanal. Así mismo, se encontró que una menor capacidad percibida para reparar los estados de ánimo negativos se relacionó con una mayor frecuencia de consumo de cannabis durante el último año. Una vez estratificados los resultados en función de los grupos de edad se observó que la capacidad percibida para reparar los estados emocionales negativos se relacionó de manera inversa con el consumo alguna vez en ambos grupos de edad, así como al consumo ante el ofreciemiento de los amigos en el grupo de 12-13 años. A su vez, una menor claridad emocional se asoció a un mayor consumo ante el ofrecimiento de los amigos en el grupo de 14-16 años. Los hallazgos de este estudio están en la línea de lo encontrado en trabajos previos que ya hallaron relación entre las bajas puntuaciones de reparación y claridad emocional y el consumo de esta sustancia. Vale la pena mencionar que sólo algunos trabajos hasta el momento han analizado los componentes separados de la IE rasgo en jóvenes y adolescentes. Sin embargo, los pocos llevados a cabo han mostrado que una menor capacidad para comprender y regular los estados emocionales se asocia a un mayor consumo de sustancias adictivas en población adolescente y universitaria (Limonero et al., 2006; Limonero et al., 2013; Ruíz-Aranda et al., 2010; Ruíz-Aranda, Fernández-Berrocal, Cabello y Extremera, 2006). Por otro lado, la presión del grupo de iguales es uno de los factores que más se asocia al inicio del consumo de drogas, así como a las situaciones de abuso (Golpe, Isorna, Barreiro, Braña y Rial, 2017; Teunissen et al., 2016). Los resultados obtenidos en esta investigación apoyan la idea de que los adolescentes con una mayor dificultad para comprender y regular las emociones podrían encontrar problemas a la hora de identificar la presión de grupo y gestionar las discrepancias entre sus motivaciones y las del resto. 
Respecto a las cuatro ramas o factores de IE habilidad se obtuvieron modelos principalmente con percepción y facilitación emocional que, en conjunto, conforman el área experiencial de IE. El área experiencial de IE se caracteriza por la habilidad para percibir y usar las emociones facilitando así la toma de decisiones y el rendimiento cognitivo de los adolescentes (Fernández-Berrocal et al, 2011). En este estudio, tanto la percepción como la facilitación emocional se asociaron de manera negativa con el consumo de cannabis alguna vez y con el consumo por ofrecimiento del grupo de amigos.

Así mismo, la habilidad de facilitación emocional se relacionó de forma negativa con la frecuencia de consumo durante el último año, así como la percepción emocional lo hizo con la cantidad de consumo semanal. Estas habilidades de IE fueron igulmente las más influyentes una vez estratificados los resultados por grupos de edad. En este sentido, percepción emocional se asoció de manera inversa tanto al consumo de cannabis alguna vez, como al consumo por ofrecimiento de los amigos en ambos grupos de edad. Por otro lado, la habilidad de facilitación emocional se relacionó de manera inversa con el consumo alguna vez en ambos grupos de edad, así como al consumo por ofrecimiento de los amigos en el grupo de 14-16 años. Estos hallazgos están en consonancia con lo encontrado por Brackett et al. (2004), en cuya investigación se observaron asociaciones negativas entre la rama experiencial de IE habilidad, el consumo de cannabis, drogas ilegales, alcohol y consumo compartido con el grupo de amigos en universitarios. En su estudio con adolescentes, Trinidad et al. (2002) también encontraron que aquellos con mayor capacidad para percibir, comprender y regular sus estados emocionales informaron de un menor consumo de tabaco y alcohol.

A pesar de la importancia que la comprensión y el manejo emocional poseen a la hora de interpretar las emociones y regularlas, estos componentes de la IE habilidad no resultaron explicativos de las variables de consumo de cannabis cuando se tuvo en cuenta la IE rasgo. Es de resaltar que, aunque las habilidades de compresión y manejo emocional no resultaron significativas en los modelos propuestos al tener en cuenta la IE rasgo, sí correlacionaron de manera inversa con algunas de las variables de consumo de cannabis. Este hecho no sólo demuestra la relevancia de conocer y procesar la información emocional sino también, de saber utilizarla para prevenir conductas de riesgo relacionadas con el consumo de cannabis a edades tempranas.

Los resultados obtenidos en este estudio amplían la evidencia previa respecto a la relación entre las variables emocionales y las conductas relacionadas con el consumo de cannabis en los jóvenes (Brackett et al., 2004; Kun et al. 2010; Limonero et al., 2006; Limonero et al., 2013; Ruíz-Aranda et al., 2010). Se podría concluir que aquellos adolescentes con un mayor repertorio de habilidades para percibir las emociones ajenas y atender las suyas propias de forma moderada, capaces de usar la información emocional para facilitar la cognición y hacer empleo de estrategias para comprender y reparar los propios estados emocionales negativos, tienen menos probabilidades de consumir cannabis. Los jóvenes con estas habilidades son más capaces de gestionar sus emociones de manera adaptativa y es posible que no necesiten utilizar otro tipo de reguladores externos para hacer frente a los acontecimientos vitales en esta etapa. En este sentido, dado que las capacidades que incluye la IE pueden ser aprendidas y mejoradas evitando conductas de riesgo para la salud (Mayer et al., 2008), consideramos que estos resultados podrían ser orientadores en el diseño de intervenciones clínicas y educativas dirigidas a prevenir la aparición de problemas de desajuste psico-social en la etapa adolescente como es el consumo de drogas, en particular el cannabis.

Nuestros resultados han de ser interpretados en el contexto de ciertas limitaciones metodológicas. En esta línea, cabe señalar que sería deseable que la muestra fuera más heterogénea incorporando así muestras de otras comunidades para poder generalizar resultados. Finalmente, es importante referirse a la naturaleza transversal del estudio. Futuros trabajos deberían continuar corroborando los resultados aquí encontrados a través de diseños prospectivos que permitan inferir relaciones de causalidad entre las variables estudiadas. A pesar de estas limitaciones, esta investigación aporta información adicional sobre la relación de la IE y el consumo de cannabis en la etapa más temprana de la adolescencia (12-16 años) y que apenas se ha estudiado. Así mismo, dado que difícilmente se encuentran investigaciones que combinen la evaluación de los constructos de IE rasgo e IE habilidad, este estudio aporta una comprensión más completa de su papel sobre el consumo de cannabis a edades tempranas.

\section{Agradecimientos}

Este estudio ha sido realizado gracias a la financiación recibida a través de la convocatoria del Programa de Personal Investigador en Formación Predoctoral de la Universidad de Cantabria (CVE-2016-11670).

\section{Conflicto de intereses}

Las autoras declaran no tener conflicto de intereses.

\section{Referencias}

Anglin, D. M., Corcoran, C. M., Brown, A. S., Chen, H., Lighty, Q., Brook, J. S. y Cohen, P. R. (2012). Early cannabis use and schizotypal personality disorder symptoms from adolescence to middle adulthood. Schizophrenia Research, 137, 45-49. doi:10.1016/j.schres.2012.01.019. 
Balluerka, N., Gorostiaga, A., Alonso-Arbiol, I. y Aritzeta, A. (2016). Peer attachment and class emotional intelligence as predictors of adolescents' psychological well-being: A multilevel approach. Journal of Adolescence, 53, 1-9. doi:10.1016/j.adolescence.2016.08.009.

Bhattacharya, M., Dutta, A. K. y Mandal, M. K. (2004). Factor structure of emotional intelligence in India. Psychological Studies, 49, 142-146.

Boden, M. T., Gross, J. J., Babson, K. A. y Bonn-Miller, M. O. (2013). The interactive effects of emotional clarity and cognitive reappraisal on problematic cannabis use among medical cannabis users. Addictive Behaviors, 38, 1663-1668. doi:10.1016/j.janxdis.2011.11.007.

Brackett, M. A., Mayer, J. D. y Warner, R. M. (2004). Emotional intelligence and its relation to everyday behaviour. Personality and Individual Differences, 36, 1387-1402. doi:10.1016/S0191-8869(03)00236-8.

Brackett, M. A. y Salovey, P. (2006). Measuring emotional intelligence with the Mayer-Salovey-Caruso Emotional Intelligence Test (MSCEIT). Psicothema, 18(Supl.), 34-41.

Claros, E. y Sharma, M. (2012). The relationship between emotional intelligence and abuse of alcohol, marijuana, and tobacco among college students. Journal of Alcohol and Drug Education, 56, 8-37.

Creemers, H. E., Dijkstra, J. K., Vollebergh, W. A., Ormel, J., Verhulst, F. C. y Huizink, A. C. (2010). Predicting life-time and regular cannabis use during adolescence; the roles of temperament and peer substance use: The TRAILS study. Addiction, 105, 699-708. doi:10.1111/ j.1360-0443.2009.02819.x.

Davis, S. K. y Humphrey, N. (2012). The influence of emotional intelligence (EI) on coping and mental health in adolescence: Divergent roles for trait and ability EI. Journal of Adolescence, 35, 1369-1379. doi:10.1016/j.adolescence.2012.05.007.

Degenhardt, L., Coffey, C., Romaniuk, H., Swift, W., Carlin, J. B., Hall, W. D. y Patton, G. C. (2013). The persistence of the association between adolescent cannabis use and common mental disorders into young adulthood. Addiction, 108, 124-133. doi:10.1111/j.13600443.2009.02819.x.

Díaz, A., Busto, A. y Caamaño, F. (2018). Alcohol, tobacco and cannabis consumption in adolescents from a multicultural population (Burela, Lugo). Adicciones, 30, 264270. doi:10.20882/adicciones.915.

Espelt, A., Bosque-Prous, M. y Marí-Dell'Olmo, M. (2019). Considerations on the use of Odds Ratio versus Prevalence or Proportion Ratio. Adicciones, 31, 257-259. doi:10.20882/adicciones.1416.

Espelt, A., Marí-Dell'Olmo, M., Penelo, E. y Bosque-Prous, M. (2017). Estimación de la Razón de Prevalencia con distintos modelos de Regresión: Ejemplo de un estudio internacional en investigación de las adicciones. Adicciones, 29, 105-112. doi:10.20882/adicciones.823.
European Monitoring Centre for Drugs and Drug Addiction (2018). European Drug Report 2018: Trends and Developments. Publications Office of the European Union, Luxembourg.

Extremera, N. y Fernández-Berrocal, P. (2006). Emotional intelligence as predictor of mental, social, and physical health in university students. The Spanish Journal of Psychology, 9, 45-5. doi:10.1017/S1138741600005965.

Fernández-Berrocal, P., Alcaide, R., Extremera, N. y Pizarro, D. A. (2006). The role of emotional intelligence in anxiety and depression among adolescents. Individual Differences Research, 4, 16-27.

Fernández-Berrocal, P., Extremera, N., Palomera, R., Ruiz-Aranda, D. y Salguero, J. M. (2011). Test de Inteligencia Emocional de la Fundación Botín para adolescentes (TIEF$B A)$. Santander: Fundación Botín.

Fernández, I., Velasco, C. y Campos, M. (2003). Inteligencia emocional, alexitimia y factores psicosociales. Encuentros en Psicología Social, 1, 246-250.

Gascó, V. P., Badenes, L. V. y Plumed, A. G. (2018). Trait emotional intelligence and subjective well-being in adolescents: The moderating role of feelings. Psicothema, 30, 310-315. doi:10.7334/psicothema2017.232.

Golpe, S., Isorna, M., Barreiro, C., Braña, T. y Rial, A. (2017). Binge drinking among adolescents: Prevalence, risk practices and related variables. Adicciones, 29, 256267. doi:10.20882/adicciones.932.

González-Yubero, S., Palomera, R. y Lázaro-Visa, S. (2019). Trait and ability Emotional Intelligence as predictors of alcohol consumption in adolescents. Psicothema, 31, 292297. doi:10.7334/psicothema2018.315.

Kun, B. y Demetrovics, Z. (2010). Emotional intelligence and addictions: A systematic review. Substance Use $\mathcal{E} \mathrm{Mi}$ suse, 45, 1131-1160. doi:10.3109/10826080903567855.

Legleye, S., Piontek, D. y Kraus, L. (2011). Psychometric properties of the Cannabis Abuse Screening Test (CAST) in a French sample of adolescents. Drug and Alcohol Dependence, 113, 229-235. doi:10.1016/j.drugalcdep.2010.08.011.

Limonero, J. T., Gómez-Romero, M. J., Fernández-Castro, J. y Tomás-Sábado, J. (2013). Influencia de la inteligencia emocional percibida y la impulsividad en el abuso de cánnabis en jóvenes. Ansiedad y Estrés, 19, 223-234.

Limonero, J. T., Tomás-Sábado, J. y Fernández-Castro, J. (2006). Perceived emotional intelligence and its relation to tobacco and cannabis use among university students. Psicothema, 18 (Supl.1), 95-100.

Lombas, A. S., Martín-Albo, J., Valdivia-Salas, S. y Jiménez, T. I. (2014). The relationship between perceived emotional intelligence and depressive symptomatology: The mediating role of perceived stress. Journal of Adolescence, 37, 1069-1076. doi:10.1016/j.adolescence.2014.07.016. 
Martins, A., Ramalho, N. y Morin, E. (2010). A comprehensive meta-analysis of the relationship between emotional intelligence and health. Personality and individual Differences, 49, 554-564. doi:10.1016/j.paid.2010.05.029.

Mayer, J. D. y Salovey, P. (1997). What is emotional intelligence? En P. Salovey y D. Sluyter (Eds.), Emotional development and emotional intelligence: Implications for educators (pp. 3-34). New York: Basic Books.

Mayer, J. D., Salovey, P. y Caruso, D. R. (2008). Emotional intelligence: New ability or eclectic traits? American Psychologist, 63, 503. doi:10.1037/0003066X.63.6.503.

Mayer, J. D., Salovey, P. y Caruso, D. (2002a). Mayer-Salovey-Caruso Emotional Intelligence Test (MSCEIT), Version 2.0. Toronto, Canada: Multi-Health Systems.

Mayer, J. D., Salovey, P. y Caruso, D. (1997). Multifactor emotional intelligence scale, student version. New Hampshire, Durham.

Míguez, M. C. y Becoña, E. (2015). Do cigarette smoking and alcohol consumption associate with cannabis use and problem gambling among Spanish adolescents? Adicciones, 27, 108-115.

Nehra, D. K., Kumar, P., Sharma, V. y Nehra, S. (2013). Alexithymia and emotional intelligence among people with cannabis dependence and healthy control: A comparative study. Dysphrenia, 5, 49-55.

Nehra, D. K., Sharma, V., Mushtaq, H., Sharma, N., Sharma, M. y Nehra, S. (2012). Emotional intelligence and self esteem in cannabis abusers. Journal of the Indian Academy of Applied Psychology, 38, 397-405.

Petrides, K. V. (2011). Ability and trait emotional intelligence. En T. Chamorro-Premuzic, S. Von Stumm y A. Furnham, (Eds.), The Wiley-Blackwell handbook of individual differences (pp. 656-678). Malden, MA: Wiley-Blackwell.

Plan Nacional sobre Drogas (2018). Encuesta sobre el uso de drogas en enseñanzas secundarias en España (ESTUDES 2016-2018). Madrid, España: Ministerio de Sanidad, Servicios Sociales e Igualdad.

Resurrección, D. M., Salguero, J. M. y Ruiz-Aranda, D. (2014). Emotional intelligence and psychological maladjustment in adolescence: A systematic review. Journal of Adolescence, 37, 461-472. doi:10.1016/j.adolescence.2014.03.012.

Rial, A., Burkhart, G., Isorna, M., Barreiro, C., Varela, J. y Golpe, S. (2019). Cannabis use among adolescents: Risk pattern, implications and possible explanatory variables. Adicciones, 31, 64-77. doi:10.20882/adicciones.1212.

Ruiz-Aranda, D., Cabello, R., Salguero, J. M., Castillo, R., Extremera, N. y Fernández-Berrocal, P. (2010). Los adolescentes malagueños ante las drogas: La influencia de la inteligencia emocional. Málaga: GEU.

Ruiz-Aranda, D., Fernández-Berrocal, P., Cabello, R. y Extremera, N. (2006). Inteligencia emocional percibida y consumo de tabaco y alcohol en adolescentes. Ansiedad y Estrés, 12, 223-230.

Saklofske, D. H., Austin, E. J., Galloway, J. y Davidson, K. (2007). Individual difference correlates of health-related behaviours: Preliminary evidence for links between emotional intelligence and coping. Personality and Individual Differences, 42, 491-502. doi:10.1016/j.paid.2006.08.006.

Salguero, J. M., Fernández-Berrocal, P., Balluerka, N. y Aritzeta, A. (2010). Measuring perceived emotional intelligence in the adolescent population: Psychometric properties of the Trait Meta-Mood Scale. Social Behavior and Personality: An international journal, 38, 1197-1209. doi:10.2224/sbp.2010.38.9.1197.

Salovey, P., Mayer, J. D., Goldman, S. L., Turvey, C. y Palfai, T. F. (1995). Emotional attention, clarity, and repair: Exploring emotional intelligence using the Trait Meta-Mood Scale. En J. W. Pennebaker (Ed.), Emotion, disclosure, and health (pp. 125-154). Washington, DC: American Psychological Association.

Schutte, N. S., Malouff, J. M., Hall, L. E., Haggerty, D. J., Cooper, J. T., Golden, C. J. y Dornheim, L. (1998). Development and validation of a measure of emotional intelligence. Personality and Individual Differences, 25, 167177. doi:10.1016/S0191-8869(98)00001-4.

Silins, E., Horwood, L. J., Patton, G. C., Fergusson, D. M., Olsson, C. A., Hutchinson, D. M. y Coffey, C. (2014). Young adult sequelae of adolescent cannabis use: An integrative analysis. The Lancet Psychiatry, 1, 286-293.

Teunissen, H. A., Kuntsche, E., Scholte, R. H., Spijkerman, R., Prinstein, M. J. y Engels, R. C. (2016). Friends' drinking norms and male adolescents' alcohol consumption: The moderating role of performance-based peer influence susceptibility. Journal of Adolescence, 53, 45-54. doi:10.1016/j.adolescence.2016.08.017.

Trinidad, D. R. y Johnson, C. A. (2002). The association between emotional intelligence and early adolescent tobacco and alcohol use. Personality and Individual Differences, 32, 95-105. doi:10.1016/S0191-8869(01)00008-3.

von Sydow, K., Lieb, R., Pfister, H., Höfler, M. y Wittchen, H. U. (2002). What predicts incident use of cannabis and progression to abuse and dependence?: A 4-year prospective examination of risk factors in a community sample of adolescents and young adults. Drug and Alcohol Dependence, 68, 49-64. doi:10.1016/S03768716(02)00102-3.

World Drug Report 2018 (United Nations publication, Sales No. E.18.XI.9).

World Medical Association. (2013). World Medical Association Declaration of Helsinki: Ethical principles for medical research involving human subjects. JAMA, 310, 2191-2194. doi:10.1001/jama.2013.281053. 